\title{
I Die Covid-19-Pandemie und Wirtschaftskrisen: die Mehrfachbelastungen von Frauen in Privathaushalten
}

\author{
Stefanie Wöhl* und Hanna Lichtenberger ${ }^{\star *}$
}

\section{Zusammenfassung}

In diesem Beitrag setzen wir die unbezahlte soziale Reproduktionsarbeit von Frauen in Privathaushalten ins Verhältnis zu bezahlter (Pflege-)Arbeit im Kontext der letzten Finanz- und Wirtschaftskrise 2008 in Spanien und der jetzigen Covid-19-Pandemie in Österreich. Dadurch werden zwei Aspekte deutlich: Erstens, welche gravierenden Konsequenzen die Einsparungen im öffentlichen Sektor seit 2010 und neue EU-Vorgaben seitdem für die Sozial- und Gesundheitssysteme haben (Spanien); zweitens, dass besonders in Krisenzeiten einer globalen Pandemie die Mehrfachbelastungen von Frauen in Privathaushalten in der sozialen Reproduktion deutlich zutage treten (Österreich).

Schlagwörter: soziale Reproduktion, Pflege, unbezahlte Arbeit, Covid-19, Finanz- und Wirtschaftskrise, Mehrfachbelastungen von Frauen

The Covid-19-Pandemic and Economic Crises: a multiple burden for women in private households

Abstract

The article discusses how unpaid social reproduction is related to paid (care) work since the latest financial and economic crisis 2008 and within the ongoing Covid-19 pandemic. We discuss this in the context of austerity measures implemented in Spain, because they show particularly well how the effects of austerity policies have an impact on the social and health system, and how women are significantly more affected by unpaid social reproductive work during the Covid-19 pandemic in a more detailed case study on Austria.

Keywords: social reproduction, care, unpaid work, Covid-19, financial and economic crisis, multiple burden of women

\footnotetext{
IIIIIIIIIIIIIIIIIIIIIIIIIIIIIIIIIII

* Stefanie Wöhl, Prof. (FH) Dr., Jean-Monnet-Professur für Diversität und soziale Kohäsion in der Europäischen Union, Fachhochschule des BFI Wien, Wohlmutstraße.22, 1020 Wien; E-Mail: stefanie.woehl@fh-vie.ac.at, Tel.: +43 1 720 12 86-28

${ }^{* *}$ Hanna Lichtenberger, Mag./MA, Universität Wien, Institut für Politikwissenschaft, Universitätsstraße 7, 1010 Wien, E-Mail: hanna.lichtenberger@univie.ac.at
} 


\section{Einleitung}

Seit den 1980er-Jahren sind viele rechtliche Regulierungen entstanden, um die Lebens- und Arbeitsbedingungen von Frauen in der Europäischen Union (EU) zu verbessern. Nach der Finanz- und Wirtschaftskrise 2008 wurden diese positiven gleichstellungspolitischen Entwicklungen jedoch durch Austeritätsprogramme in den meisten Mitgliedstaaten konterkariert. Heute, in der Covid-19-Krise einer globalen Pandemie, zeigen sich diese Einsparungen oder Privatisierungen im öffentlichen Sektor des Sozial- und Gesundheitsbereiches besonders signifikant, wenn in einigen Mitgliedstaaten $\mathrm{zu}$ wenige Krankenhausbetten für IntensivpatientInnen vorhanden sind oder MitarbeiterInnen von privaten Pflegeheimen nicht mehr zur Arbeit gehen und verstorbene Menschen dort unbetreut vorgefunden werden, wie es in Spanien im Frühjahr 2020 der Fall war (Wandler 2020).

Dass Frauen zudem z. B. durch Arbeitsplatzverluste in Krisenzeiten mehr unbezahlte Arbeit in der sozialen Reproduktion leisten, wurde in vielen internationalen Studien bereits belegt (Annesley/Scheele 2011; Elias/Gunarwardena 2013; DG Justice 2013; Horn 2009; Karramessini/Rubery 2014; Kurz-Scherf/Scheele 2013; Kantola/Lombardo 2017). In diesem Beitrag möchten wir deshalb die unbezahlte soziale Reproduktionsarbeit in Privathaushalten ins Verhältnis zu bezahlter (Pflege-)Arbeit in den Kontext der letzten Finanz- und Wirtschaftskrise 2008 und der jetzigen Covid-19-Pandemie setzen. Dabei werden zwei Aspekte deutlich: Erstens, welche gravierenden Konsequenzen die Einsparungen im öffentlichen Sektor seit 2010 und die neuen EU-Vorgaben im Rahmen des Europäischen Semesters seitdem auf die Mitgliedstaaten haben (Bruff/Wöhl 2016); zweitens, dass die „strukturelle Sorglosigkeit“ (Aulenbacher/Dammayr 2014: 68) des Kapitalismus in Krisenzeiten besonders deutlich zutage tritt und Frauen unter den Folgeeffekten von Krisen noch mehr unbezahlte Arbeit leisten.

Im Folgenden werden dafür zuerst die Auswirkungen von einigen supranationalen EU-Austeritätsmaßnahmen auf die Privathaushalte und Sozialsysteme anhand von Spanien kursorisch dargestellt. Der Fokus auf Spanien ist dadurch begründet, dass dort massiv Sozialausgaben im Gesundheitsbereich gekürzt wurden und die Arbeitslosigkeit zwischen 2008 und 2015 stark angestiegen ist. Spanien ist zudem eines der Länder, die nach 2010 Gelder aus dem Europäischen Stabilitäts-Mechanismus (ESM) erhielten.
Diese Entwicklungen werden ins Verhältnis gesetzt zur derzeitigen Covid-19-Pandemie. Anhand des Fallbeispiels Österreich wird anschließend durch neuere Studienergebnisse ausführlicher gezeigt, welche Auswirkungen die Covid-19-Pandemie speziell auf Frauen und Privathaushalte bisher hat, sowohl im entlohnten Pflege- und Betreuungssektor in Privathaushalten als auch in der sogenannten unbezahlten sozialen Reproduktionsarbeit, wie kochen, putzen, waschen, betreuen und pflegen in Privathaushalten. Auch hier kann festgehalten werden, dass die bestehende geschlechtsspezifische soziale Reproduktionsarbeit weiterhin mehrheitlich von Frauen unentgeltlich ausgeübt wird und es eher zu einer Intensivierung der ungleichen Verteilung von sozialer Reproduktionsarbeit im Privathaushalt kommt (Derndorfer et al. 2021; Six et al. 2021). Zudem sind auch in Österreich in den letzten Jahren Einsparungen bei Intensivbetten vorgenommen worden und der bezahlte Pflegesektor wurde nicht ausgebaut bzw. ist nach wie vor ein niedrig entlohnter Sektor, in dem entsprechend viele Frauen arbeiten. Überdies weist gerade der bezahlte als auch der unbezahlte Pflegesektor verschiedene Schieflagen auf, die entlang der Kategorien Klasse, Geschlecht, Alter, StaatsbürgerInnenstatus und weiterer Merkmale sozialer Differenzierung verlaufen, wie wir am Beispiel der 24-Stunden-Betreeuung in Österreich verdeutlichen werden (Lichtenberger/Wöhl 2020; Wöhl 2021).

\section{Soziale Reproduktion, Austeritätsmaßnahmen und die ,strukturelle Sorglosigkeit des Kapitalismus}

Brigitte Aulenbacher und Maria Dammayr argumentieren, der Kapitalismus sei durch eine „strukturelle Sorglosigkeit“ (Aulenbacher/Dammayr 2014: 68) geprägt, in der es zur Unterordnung der Sorgearbeit und somit auch der sozialen Reproduktion insgesamt unter die Erfordernisse des Marktes kommt. ,Soziale Reproduktion' erfasst begrifflich sowohl die unentgeltliche Pflege und Betreuung von Angehörigen, als auch unbezahlte Hausarbeit sowie sozialstaatliche Fürsorgeleistungen im bezahlten Pflegesektor (Bakker/Gill 2003). Die oben erwähnte Unterordnung realisiert sich dabei in drei Formen: erstens durch die Verschiebung von Sorgeerfordernissen in den privaten Bereich, wo dies in ausreichendem Maße möglich ist; zweitens durch Investitionen dort, wo Rendite in Gegenwart und Zukunft zu erwarten sind; drittens bestehe ein Aspekt dieser Unterordnung in der Inwertsetzung der Sorge, etwa durch Privatisierungen im Gesund- 
heitssektor (Aulenbacher 2020: 396-397). Die Sorglosigkeit des Kapitalismus zeige sich dadurch, dass die Sorgearbeiten „in diesem Falle anderen Zwecken unterworfen und somit nicht primär nach Maßgabe ihrer Lebensdienlichkeit organisiert werden“" (Aulenbacher/Bachinger/Deciéux 2015: 7). Die strukturelle Sorglosigkeit des Kapitalismus wird mit unterschiedlicher Ausprägung je nach Wohlfahrtsstaatsmodell „klassen-, geschlechts- und ethnizitätsbasiert institutionell und individuell lebbar gemacht" (Aulenbacher/ Bachinger/Deciéux 2015: 8), wodurch sich nationale und globale Ungleichheiten ergeben. Innerhalb der globalen politischen Ökonomie wird die unbezahlte soziale Reproduktionsarbeit in Privathaushalten in der internationalen Arbeitsteilung immer noch vorausgesetzt, und auch die Weltbank hat erst nach massivem Lobbying soziale Reproduktionsarbeit als Politikfeld auf ihre politische Agenda gesetzt (Bakker 2007). Das bedeutet, dass sowohl supranationale Politiken und AkteurInnen sowie nationalstaatliche Politiken und das jeweilige Geschlechterarrangement und Geschlechterregime eines Staates bei der Verteilung von bezahlter als auch unbezahlter Arbeit eine bedeutende Rolle spielen.

In den Mitgliedstaaten der EU gibt es, wenn auch in unterschiedlicher Ausprägung, Systeme sozialer Sicherung in Form von Sozialstaaten vor allem in den Ländern West- und Nordeuropas sowie einen vorhandenen öffentlichen Sektor. Aber auch in der EU gab es eine Verschiebung von unbezahlter Arbeit nach der Finanzkrise 2008 innerhalb von Privathaushalten zuungunsten von Frauen, wie bereits seit Finanzund Wirtschaftskrisen in Asien und Lateinamerika bekannt ist (Benería 1991; Herrera 2012; Horn 2009; Elias/Gunarwardena 2013; Elson 2012; Kantola/Lombardo 2017; Karramessini/Rubery 2014; Wöhl 2017; Young 2003).

„Households often adjust in reaction to economic downturn by using unpaid labour to replace services that had been purchased in markets before the crisis" (Fukuda-Parr et al. 2013: 22).

Mehrfachbelastungen und psychischer Druck auf die einzelne Person und vor allem auf Frauen ist daher ein gemeinsames Merkmal in ökonomischen und pandemiebedingten Krisenzeiten. Auf diese bemerkenswerte Kontinuität, dass bestehende Ungleichheiten in den Geschlechterverhältnissen Krisen ,stabilisieren und gleichzeitig Krisen vergeschlechtlichte Ungleichheiten verschärfen, weisen auch Veronika Duma und Katharina Hajek (2014) in ihrem historischen Ver- gleich zwischen der Wirtschaftskrise in Österreich nach dem Zusammenbruch der Creditanstalt 1929 f. und der Finanz- und Wirtschaftskrise 2008f. hin. In den Jahren seit 2008 wurden in der EU zudem finanzund wirtschaftspolitische Steuerungsmaßnahmen auf nationaler und supranationaler Ebene durchgesetzt, die vor allem die Mitgliedstaaten zu Austeritätsmaßnahmen im staatlichen öffentlichen Sektor anhalten sollen, um weitere Staatsverschuldungen $\mathrm{zu}$ vermeiden und den Stabilitäts- und Wachstumspakt von 1996 weiterhin einhalten zu können (Klatzer/ Schlager 2020). Ziel der umfangreichen Regelungen ist, die Nationalstaaten auf ausgeglichene öffentliche Finanzen zu verpflichten, wie zum Beispiel mit dem sogenannten Fiskalvertrag, der Schuldenobergrenzen für die Verschuldung eines Landes in Relation zu seinem BIP festlegt. Der Fokus der rechtlichen Vorgaben ist dabei auf die Kürzung von Staatsausgaben gerichtet, indem Pensionen, Gesundheitskosten und Sozialausgaben gesenkt werden. In den verschiedenen Mitgliedstaaten wurden zudem jegliche Maßnahmen, die Gender Budgeting und somit die Folgewirkungen für die Geschlechterverhältnisse beachtet hätten, bisher nicht umgesetzt (O'Hagan/Klatzer 2018; Klatzer/Schlager 2014). Da der Staat nur im öffentlichen Sektor einsparen kann, waren vor allem die öffentliche Gesundheitsversorgung und weitere sozialstaatliche Leistungen von diesen Einsparungen in der Vergangenheit betroffen - Bereiche, in denen vor allem Frauen arbeiten. Obwohl die EU sich zu Gender Mainstreaming und Gender Budgeting verpflichtet hat, wurden die fiskalpolitischen Regulierungen nicht auf ihre Folgewirkungen für Männer* und Frauen* oder für Frauen* besonders relevante Arbeitsmarktsektoren hin überprüft. Auch im „Europäischen Semester“, das seitens der EU-Kommission die nationalen Haushaltsbudgets und Defizitgrenzen der Mitgliedstaaten innerhalb eines Jahreszyklus überwachen soll, wurde zuletzt vom Europäischen Institut für Gleichstellungspolitik (EIGE) festgestellt, dass überhaupt keine GenderBudgeting-Maßnahmen implementiert wurden (European Institute for Gender Equality 2018: 24). Dies ist besonders für Mitgliedstaaten relevant, die stark von der Finanz- und Wirtschaftskrise nach 2008 betroffen waren und höhere Staatsverschuldungen hatten, wie am Beispiel von Spanien besonders deutlich wird.

Spanien war vor dem Ausbruch der Finanz- und Wirtschaftskrise 2008 eine schnell wachsende Ökonomie mit hohen durchschnittlichen BIP-Wachstumsraten von 3,7 Prozent in den Jahren zwischen 
1995 und 2007 (López/Rodríguez 2011). Aufgrund dieser Wachstumsraten und einem hohen Anteil von Arbeitsimmigration vor 2008 boomte auch die Immobilienbranche, die später die größten Jobverluste verzeichnete. Spanien beantragte 2011 Hilfsgelder von der EU, um den Bankensektor zu stabilisieren, der aufgrund von Privatverschuldungen durch Immobilien-Kredite instabil geworden war und einen damit zusammenhängenden starken Wirtschaftseinbruch im Jahr 2009 verursachte. Da besonders der Bankensektor stark eingebrochen war, geriet der Staat mit seinen Bankenrettungsmaßnahmen selbst unter Druck der Finanzmärkte. Einkommensschwache Privathaushalte traf es in der Folge am meisten, wenn Arbeitsplatzverluste stattfanden, Delogierungen aufgrund von Zwangsräumungen erfolgten und mehr Frauen unbezahlte Arbeit im Haushalt übernahmen (Herrera 2012; Lombardo/León 2015). Die hohe Kreditverschuldung der Privathaushalte führte dazu, dass viele spanische Familien ihre Häuser verlassen mussten, da sie ihre Kredite nicht mehr bedienen konnten (Huke/ Wöhl 2018). Gleichzeitig wurden Steuern erhöht und große Einsparungen vorgenommen, die meisten betrafen dabei den Bildungs- und Gesundheitssektor (Lombardo 2017; Lombardo/León 2015). Spanien hat zudem einen großen privaten Sektor in der Gesundheitsversorgung, was vor allem in der jetzigen Covid19-Pandemie zu einem massiven Problem wurde, da es schwierig war, die öffentliche Gesundheitsversorgung mit entsprechenden Intensivbetten überhaupt aufrechtzuerhalten (Wandler 2020). So kam es schließlich dazu, dass private Spitäler im März 2020 unter staatliche Kontrolle gestellt wurden.

Spanien wird zwar als sozialstaatliches Doppelverdiener-Modell angesehen, sodass zumeist beide (Ehe-) PartnerInnen jeweils einer Lohnarbeit nachgehen, das System sozialer Sicherung hält jedoch Vollzeitbeschäftigung als Norm aufrecht, um volle Sozialleistungen empfangen zu können, sodass Frauen in Teilzeitarbeit besonders benachteiligt werden (González Gago/ Kirzner 2014; Serrano/González Gago 2013; Lux/ Wöhl 2015). Die geschlechtsspezifische Arbeitsteilung hat sich zudem nur langsam verändert: Männer sind häufiger in bezahlten Arbeitsverhältnissen und haben mehr Freizeit, während Frauen eher in kurzzeitigen Vertragsverhältnissen und in Teilzeitarbeit anzutreffen sind. Sie übernehmen auch weiterhin die meiste unbezahlte soziale Reproduktionsarbeit (Lombardo 2017; Farré et al. 2020). Wie jüngste Studien belegen, hat sich die Verteilung von unbezahlter Haus- und Pfle- gearbeit von Frauen in der Covid-19-Pandemie weiter zu ihren Ungunsten verschoben, obwohl spanische Männer im ersten Lockdown auch unbezahlte Hausarbeiten übernahmen, als die Schulen im Frühjahr 2020 geschlossen wurden und es vermehrt zu HomeofficeArbeit kam (Farré et al. 2020). Davon profitierten aber hauptsächlich besser gebildete Haushalte, wie auch international vergleichende Studien belegen (Alon et al. 2020; Adams-Prassl et al. 2020). Zudem konnten Beschäftigte in systemrelevanten Sektoren nicht gleichermaßen Kinderbetreuung zu Hause leisten, und Beschäftigte, die keinen Universitätsabschluss haben, wurden während des ersten Lockdowns eher von der Lohnarbeit beurlaubt als andere, besser ausgebildete Beschäftigte (Farré et al. 2020: 15).

Löhne im öffentlichen (Gesundheits-)Sektor wurden zudem seit der Finanzkrise 2008 bereits um 5 Prozent gekürzt. Außerdem wurde das Mindesteinkommen nicht an die Inflation angepasst (Lombardo 2014; Lombardo/Alonso 2020). Zudem gab es Verschlechterungen im Bereich der Pflege von Angehörigen und Kindern, was ursprünglich in einem Gesetz von 2006 geregelt wurde. Seit 2010 wurden hier durch umgesetzte Austeritätsmaßnahmen aber Zuzahlungen gekürzt und der Sozialversicherungsschutz für pflegende Angehörige gestrichen (Lombardo/León 2015; Lombardo/Alonso 2020). Kosten für Arzneimittel und für medizinische Behandlungen müssen zum Teil von den Betroffenen selbst übernommen werden. Die jetzige Covid-19-Pandemie traf also sowohl auf ein bereits seit der Finanz- und Wirtschaftskrise von 2008 geschwächtes Sozial- und Gesundheitssystem als auch auf eine verminderte Einkommenssituation von Teilen der Bevölkerung und auf Mehrfachbelastungen von Frauen. Die Folgen der Privatisierungen führten dazu, dass das Gesundheitssystem in Spanien 2020 durch die Covid-19-Pandemie schnell überlastet war.

Im nächsten Abschnitt soll am Fallbeispiel von Österreich detaillierter gezeigt werden, wie vor allem Frauen und Privathaushalte durch die Covid-19-Krise bisher betroffen sind und warum Mitgliedstaaten, die nach der Finanzkrise 2008ff. nicht im gleichen Ausmaß auf Austeritätspolitik setzten, im Gesundheitsbereich besser auf die jetzige Mehrfachkrise der Pandemie reagieren konnten. Gleichzeitig setzen aber auch Länder wie Österreich dennoch die unbezahlte soziale Reproduktionsarbeit von Frauen in Privathaushalten voraus und haben zugleich den bezahlten öffentlichen oder privaten Pflegesektor bisher zu wenig gefördert. 


\section{2. Österreich: unbezahlte und bezahlte Arbeit in der Pflege und Betreuung während der Covid-19- Pandemie}

Trotz kurzzeitiger Aufmerksamkeit für die Arbeitsbedingungen, insbesondere im Bereich der Pflege und Betreuung und die damit verbundene Belastung von Eltern, zeigt die Covid-19-Pandemie in Österreich, welche geringe Anerkennung bezahlte oder unbezahlte Pflege und Betreuung von Angehörigen/Pflegebedürftigen und Kindern und jene Bereiche der (systemrelevanten) Erwerbsarbeit, in denen Frauen überpräsent sind, haben. Wie schon die Finanz- und Wirtschaftskrise 2008f. bedeutet die Covid-19-Pandemie eine Verschärfung von Reproduktionsgefährdungen, die sich zu Reproduktionskrisen verdichten. Die Covid19-Pandemie führte $\mathrm{zu}$ einer massiven Neuordnung von Arbeits- und Sorgearrangements und zu einer Familialisierung von Sorgearbeit. Die im Folgenden erwähnten empirischen Studien zeigen bisher alle, dass es zu einer Retraditionalisierung der bestehenden Arbeitsteilung zwischen Männern und Frauen in heterosexuellen Kleinfamilien kam (Allmendinger 2021). Die Folgen der „Sorglosigkeit des Kapitalismus“ (Aulenbacher/Dammayr 2014) treten aber auch im Bereich der kommodifizierten sozialen Reproduktion zutage, wie im Folgenden anhand einiger Beispiele gezeigt werden soll. Wir erkennen aktuell zumindest drei grobe Tendenzen im Bereich der sozialen Reproduktion und Pflege:

1. Erstens griff die Krisenbearbeitung der von ÖVP und Grünen zusammengesetzten Regierungskoalition im Bereich der Pflege umfassend auf Frauen und ihre unbezahlte Arbeit in Privathaushalten zurück, etwa in ihrer Rolle als Mütter oder als pflegende Angehörige, um im Kontext der Pandemie während der Lockdowns entstandene Sorgelücken zu füllen (Derndorfer et al. 2021).

2. Fehlende Ressourcen, belastende Arbeitsbedingungen in der kommodifizierten Pflege- und Betreuungsarbeit, die auch im Kontext der Austerität zu verstehen sind, treffen Frauen stark und ihre Folgen werden etwa in der 24-Stunden-Betreuung klassenspezifisch externalisiert; zudem setzte die Krisenpolitik Frauen als Arbeitskraft in systemrelevanten Bereichen einem verstärkten Erkrankungsrisiko aus - ohne von Beginn an ausreichend Schutzmaßnahmen zu treffen (Lichtenberger/Wöhl 2020).

3. Die Covid-19-Pandemie führte in unterschiedlichen Monaten seit März 2020 bis Mai 2021 auch zu einem Rückgang der Lohnarbeit von Frauen, wodurch sich langfristig ungleiche Sorgearrangements in den Privathaushalten vertiefen könnten und Arbeitslosigkeit von Frauen ein Problem darstellt (AMS 2021).

\subsection{Bezahlte Soziale Reproduktionsarbeit in der Covid-19-Pandemie}

Viele Bereiche der Wirtschaft, die schon zu Beginn der Krise als systemrelevant verstanden wurden und persönlichen Kontakt zu anderen Menschen (KundInnen, KlientInnen, Kinder) erfordern, sind stark von Frauenerwerbstätigkeit geprägt: Krankenhäuser, stationäre und mobile Pflege und Betreuung, der Lebensmitteleinzelhandel, Kindergärten und Schulen (Statistik Austria 2019; Wirtschaftskammer Österreich 2019). Trotz der hohen gesellschaftlichen Bedeutung dieser Bereiche gehören etwa der Handel oder haushaltsbezogene Betreuungstätigkeiten zu den schlecht bezahlten Berufszweigen in Österreich. Die kurzzeitige Aufmerksamkeit für die Arbeitsbedingungen im Handel oder der Pflege führte jedoch kaum zu strukturellen Veränderungen oder höherer Entlohnung. Vielmehr verdeutlichte die Corona-Pandemie strukturelle Problemlagen insbesondere im Bereich der Pflege und verschärfte diese zusätzlich.

2019 waren rund 63.000 Menschen im Bereich der Pflege und Betreuung von älteren Menschen und Menschen mit Behinderungen erwerbstätig. Angesichts der demografischen Entwicklung und der veränderten Familienstrukturen steigen weibliche Beschäftigungszahlen in diesem Sektor insgesamt stark an, insbesondere im Bereich der stationären Pflege und Betreuung. Dort beträgt der Frauenanteil der Beschäftigten 81 Prozent, in der mobilen Pflege sogar 93 Prozent (Bauer 2019). Strukturelle Probleme des Sektors sind etwa die geringe Entlohnung, die langen Arbeitszeiten und die Tendenz zur Taylorisierung der Pflege und Betreuung. Daraus resultiert, dass die Unzufriedenheit im Sektor besonders groß ist. Ein Viertel der Beschäftigten im Gesundheitsbereich denkt mindestens einmal im Monat darüber nach, den Job zu wechseln, und knapp die Hälfte der Beschäftigten in der stationären Pflege gibt an, die Arbeitsbedingungen hätten sich in den letzten Jahren verschlechtert (Bauer et al. 2018: 70; Arbeiterkammer 2019).

Im Zuge der Covid-19-Pandemie stieg die Arbeitsbelastung der KollegInnen in der stationären Pflege und Betreuung durch den Wegfall der Hilfe und der sozialen Kontakte durch pflegende Angehörige, 
weil die Einrichtungen isoliert wurden. Im mobilen Bereich kam es, oft aus Angst vor einer Ansteckung, zur Reduktion der mobilen Pflege und Betreuung durch die KlientInnen oder die Angehörigen, wodurch es in diesem Bereich sogar zur Anmeldung von Kurzarbeit kam (Bina/Magnus 2020: 25). Gleichzeitig wurde vielerorts der Pflegeschlüssel erhöht, um die Arbeit in gleichbleibenden Teams zu ermöglichen. Insbesondere für die mobilen Pflege- und Betreuungskräfte, aber auch im stationären Bereich fehlten anfangs Schutzkleidung (insb. Schutzbrillen, Schutzmäntel, $\mathrm{FFP}_{2}$ - oder $\mathrm{FFP}_{3}$-Masken), um Beschäftigte und KlientInnen ausreichend zu schützen (u.a. Bina/ Magnus 2020: 25). Dort, wo Schutzkleidung und Masken vorhanden waren, klagten die Beschäftigten im Pflege- und Betreuungsbereich über die schlechte Qualität der Produkte und/oder über eine höhere körperliche Belastung, etwa weil das Atmen durch $\mathrm{FFP}_{3}$-Masken schwerer fällt (Fikenzer et al. 2020). Besonderen Belastungen waren die 24-StundenBetreuerInnen ausgesetzt, die ihre Dienstzeiten in Österreich zum Teil um Monate verlängern mussten und damit ohne Erholung drei Monate am Stück arbeiteten. 2018 wurden etwa sechs Prozent der österreichischen PflegegeldbezieherInnen durch zumeist aus angrenzenden EU-Staaten kommende 24-Stunden-PersonenbetreuerInnen versorgt (Nagl-Cupal et al. 2018), konkret waren 201961.989 selbstständige PersonenbetreuerInnen bei der österreichischen Wirtschaftskammer registriert, davon waren 94 Prozent Frauen. Über 98 Prozent der PersonenbetreuerInnen verfügten 2019 nicht über die österreichische StaatsbürgerInnenschaft (Schober 2020). Diese Form der „Rund-um-die-Uhr“-Betreuung stellt einen Lückenschluss im österreichischen Pflegesystem dar, welcher ohne diese transnationalen „Pflegeketten“ mit vergleichbarem Kostenaufwand nicht anders zu realisieren wäre. Schon vor der Pandemie stellte diese Form der Erwerbsarbeit ein Arbeitsverhältnis dar, das nur durch Sonderregelungen und Scheinselbstständigkeit vor dem österreichischen Arbeitsschutzgesetz bestehen kann und in besonderem Maße auf die rassistisch konnotierte Externalisierung von Sorgearbeit und auf die Umgehung von Sorgekonflikten über eine transnationale Bearbeitung verweist (Aulenbacher et al. 2015: 6-7). Mehrfach wurden seit Beginn der Corona-Pandemie in Österreich die Ein- und Ausreisebestimmungen in unterschiedlichem Ausmaß beschränkt - was kurzfristig zu Versorgungsproblemen in der Personenbetreuung führte.
2.2 Unbezahlte Arbeit von Frauen als Krisenbewältigungsstrategie

Anhand zweier Bereiche der privaten sozialen Reproduktionsarbeit wird im Folgenden verdeutlicht, wie die Covid-19-Maßnahmen in besonderem Maße zur Retraditionalisierung von vergeschlechtlichter Arbeitsteilung und der Belastung von Frauen führten: einmal in der Angehörigenpflege und einmal in der Sorgearbeit von Familien mit Kindern.

\subsubsection{Frauen mit Kindern in der Covid-19-Pandemie}

Während im späteren Verlauf der Pandemie über die Bedeutung von Kindern und Jugendlichen am Infektionsgeschehen kontrovers diskutiert wurde, waren die Umstellung der Schulen auf Distance Learning/Homeschooling und die maximale Ausdünnung in den Kindergärten zwei wichtige Maßnahmen zur Eindämmung des Infektionsgeschehens im ersten Lockdown ab März 2020. Diese Maßnahmen hatten weitreichende Folgen für österreichische Familien. Ein Rechtsanspruch auf eine Sonderbetreuungszeit war ebenso nicht gegeben wie die Deckung der vollen Zeit der Maßnahmen. Vorausgesetzt wurde, dass jene, die im Homeoffice arbeiten können, die Lernbetreuung und andere Sorgearbeiten nebenbei übernehmen konnten. Eine Verstärkung der Mehrfachbelastung von Frauen war die Folge.

Die Politik der österreichischen Bundesregierung scheint von der Annahme geprägt, dass Kinderbetreuung zu Hause keine Arbeit ist - nur so kann angenommen werden, dass Homeoffice und Kinderbetreuung miteinander vereinbar wären. Dass dem nicht so ist, zeigt das Ergebnis einer SORA-Studie zu der ersten Phase des Lockdowns: Jeder fünfte befragte Elternteil arbeitete am Höhepunkt der Covid-19-Krise häufiger frühmorgens, abends oder am Wochenende, jeder Zehnte sogar nachts, um trotz Kinderbetreuung Zeit für Erwerbsarbeit zu finden (SORA 2020). Dies verschlimmerte sich sogar im zweiten Lockdown im Herbst 2020, als Mütter noch häufiger zu Randzeiten ihrer Erwerbsarbeit nachgingen, während der Anteil bei den Männern, die ihre Erwerbsarbeit in den frühen Morgenstunden, abends oder nachts erledigten, wieder sank (SORA 2020).

Eine Studie des Austrian Corona Panel Project (ACPP) an der Universität Wien zeigte für die erste Phase des Lockdowns im Frühjahr 2020, dass die Covid-19-Pandemie bei der Hälfte der befragten Familien (51 Prozent) einen Work-Family-Konflikt ergab 
(Berghammer 2020a). Ob und wie häufig es zu einem Vereinbarkeitskonflikt kam, unterschied sich wesentlich nach Familien-, Arbeits- und Wohnsituation. Besonders betroffen waren Personen mit Kindern (61 Prozent). Das Alter der Kinder spielte dabei eine große Rolle: Die Häufigkeit eines Work-Family-Konflikts war tendenziell höher mit Kindern im Kleinkind- und Vorschulalter als mit Schulkindern, weil diese insgesamt mehr Betreuungszeit benötigen und diese oft weniger gut plan- und eingrenzbar ist. Dementsprechend gibt in der SORA-Studie fast die Hälfte (46 Prozent) der befragten Eltern an, dass die Situation sie sehr stark belastete (SORA 2020). Im zweiten Lockdown im Herbst 2020 war auch eine signifikant höhere Rate an Kindern im Kindergartenalter festzumachen, die die Bildungseinrichtung trotz des Lockdowns besuchten (SORA 2020).

Im Bereich der Kinderbetreuung zu Hause waren es vor allem die Mütter, die ihre Kinder betreuten und mit ihnen lernten: 47 Prozent der Frauen, aber nur 9 Prozent der Männer wendeten viel mehr Zeit für diese Tätigkeiten auf. Die Doppelbelastung von Frauen nahm markant zu: Es zeigt sich, dass Mütter in Vollzeiterwerbstätigkeit in der ersten Phase der Covid-19Krise doppelt so häufig (63 Prozent) viel mehr Zeit mit Kinderbetreuung verbrachten als Väter (30 Prozent) (Berghammer 202ob). Die ForscherInnen kommen zu dem Schluss, dass die Arbeitsteilung in Familien mit Kindern in der Covid-19-Pandemie noch traditioneller geworden ist. Auch wurde von Frauen zusätzlich deutlich mehr Zeit für Hausarbeit aufgewendet: 16 Prozent der Frauen, aber nur 9 Prozent der Männer gaben in der Umfrage an, mehr Zeit für Hausarbeit aufgebracht zu haben. Interessant ist hierbei auch die vergeschlechtlichte Wahrnehmung der ungleichen Verteilung von sozialer Reproduktionsarbeit.

Die Studie von SORA zu den Auswirkungen des zweiten Lockdowns im Herbst 2020 verweist darauf, dass es mehr Männer gebe, die angeben, sich die Kinderbetreuung gleichermaßen mit dem oder der PartnerIn aufzuteilen als Frauen in Österreich. Dieser Umstand ist kein österreichisches Spezifikum, zeigt doch eine Umfrage des Marktforschungsunternehmens Ipsos im Auftrag der Bertelsmann Stiftung für Deutschland, dass zwar 66 Prozent der Männer angaben, sich die Aufgaben der Kinderbetreuung und Hausarbeit gerecht $\mathrm{zu}$ teilen, aber weniger als jede zweite Frau entsprechende Angaben machte (von Würzen 2020: 2). 49 Prozent der Frauen gaben in dieser Umfrage zudem an, die Belastung sei im Moment körperlich und psychisch zu hoch (von Würzen 2020: 2). In eine ähnliche Richtung weist auch eine Befragung von CARE mit 10.00o Frauen aus 38 Ländern. Global gaben 27 Prozent der Frauen, aber nur 10 Prozent der Männer an, dass sie durch die Covid-19-Pandemie in ihrer mentalen Gesundheit eingeschränkt wurden (Janoch 2020).

Die Covid-19-Familienpolitik schlägt sich auch im Erwerbsleben von Frauen nieder: Auf der einen Seite ist das Erwerbsausmaß von Müttern in der ersten Phase des Lockdowns im Frühjahr 2020 im Durchschnitt stärker zurückgegangen als jenes von Vätern. Zudem waren Frauen in der Covid-19-Pandemie im Zeitraum von März bis November 2020 stärker von Arbeitslosigkeit betroffen als Männer, was sich vor allem aus den Dienstleistungsbranchen wie Tourismus, Reinigung und Handel ergibt, in denen mehr Frauen als Männer tätig sind (AMS 2021). Das sind jene Branchen, die nicht auf Homeoffice umstellen konnten und daher höhere Kurzarbeits- und Arbeitslosenzahlen verzeichneten. Auf der anderen Seite sind aber überproportional viele Frauen in als systemrelevant verstandenen Branchen tätig, die persönlichen, körpernahen Kontakt zu KundInnen, KlientInnen oder Kindern erfordern: Krankenhäuser, stationäre und mobile Pflege und Betreuung, Lebensmitteleinzelhandel, Kindergärten und Schulen (Statistik Austria, 2019; Wirtschaftskammer Österreich, 2019).

\subsubsection{Frauen als pflegende Angehörige}

2019 gab es in Österreich rund 466.360 PflegegeldbezieherInnen. Geschätzt wird, dass rund 80 Prozent zu Hause betreut werden - mehrheitlich von pflegenden Angehörigen, die wiederum fast ausschließlich Frauen sind. Das österreichische Pflegegeldsystem hat einen starken Fokus auf individuelle Wahlfreiheit der pflegebedürftigen Menschen, es baut implizit und explizit auf Verfügbarkeit von meist weiblichen Angehörigen und deren kostenlose Pflegearbeit im Privathaushalt auf und fördert somit den Ausstieg aus der regulären Erwerbsarbeit für pflegende Angehörige. Gleichzeitig ist das Leistungsniveau im Pflegegeld-Gesetz so gering, dass eine bedarfsgerechte professionelle Pflege für Privathaushalte nicht finanzierbar ist, die über ein geringes Einkommen oder geringe Pensionen verfügen. Daher unterstützen mobile Dienste nur bei 25 Prozent der Gepflegten im Privathaushalt zusätzlich (Nagl-Cupal et al. 2018: 39).

Entlastungsangebote wie Tagesbetreuungszentren, Hilfe durch NachbarInnen, Betreuungsstunden von
IIIOMENTUM QUARTERLY IIIIIIIIIIIIIIIIII 125 
Heimhilfen/PflegeassistentInnen, aber auch Therapien (bspw. Ergo- oder Physiotherapien) fielen durch den Lockdown z. T. weg. Pflegende Angehörige wurden im Bereich der Versorgung mit Schutzkleidung und Pflegehilfsmitteln der sich zuspitzenden Versorgungssituation und der Preisentwicklung am freien Markt überlassen, erst zum Jahreswechsel 2021 erhielt die Gruppe der über 65-Jährigen zehn Stück FFP2-Masken von der Bundesregierung per Post zugestellt. Der für pflegende Angehörige von der Regierung erst nachträglich vorgesehene Sonderbetreuungsurlaub umfasste lediglich drei Wochen, Rechtsanspruch darauf gab es keinen. Die Volkshilfe Österreich veröffentlichte im Sommer 2020 eine nichtrepräsentative, ${ }^{1}$ aber dennoch beachtenswerte Befragung von 100 pflegenden Angehörigen von armutsbetroffenen Demenzerkrankten (Volkshilfe Österreich 2020). Auch wenn diese Gruppe mehrdimensionalen Benachteiligungen unterliegt, so lassen sich dennoch Tendenzen der Belastung von pflegenden Angehörigen durch die Covid-19-Pandemie ablesen. 78 der 100 Befragten gaben für die erste Phase des Lockdowns im Frühjahr 2020 an, dass sie durch die Corona-Pandemie mehr Betreuungsstunden geleistet hatten. Dadurch ergab sich für 16 der 100 Angehörigen die Notwendigkeit, das Ausmaß der Erwerbstätigkeit ganz oder zum Teil verändern zu müssen, um mehr Zeit für die pflegebedürftigen Angehörigen zu haben - wobei anzumerken ist, dass bereits 27 Prozent der Befragten selbst in Pension waren. 66 Befragte gaben an, sich oft (31 Prozent) oder zumindest hin und wieder (35 Prozent) mit der Pflege und Betreuung überfordert gefühlt zu haben. Von jenen, die eine/n 24-StundenPersonenbetreuerIn eingestellt hatten, erlebten 65 Prozent jene Situation, als die Grenzen im Frühjahr 2020 geschlossen wurden als sehr belastend. Gleichzeitig waren pflegende Angehörige in Haushalten, in denen die 24-Stunden-BetreuerInnen während des Lockdowns die gesamte Zeit im Haushalt verblieben, grundsätzlich weniger belastet durch die Covid-19-Pandemie als andere pflegende Angehörige (Volkshilfe Österreich 2020).

$1 \quad 100$ abgeschlossene Gespräche $(\mathrm{n}=100)$ im Zeitraum vom 25. Mai bis zum 3. August 2020; durchgeführt von Mitarbeiterinnen der Volkshilfe Österreich; Kontaktdaten aus den positiv bearbeiteten Anträgen des Fonds Demenzhilfe Österreich aus den Jahren 2019 und 2020. Im Zuge der Mittelvergabe wurde die Einkommenssituation der AntragstellerInnen im Hinblick auf die Armuts- und Ausgrenzungsbetroffenheit geprüft. Die Datenauswertung wurde von den Verfasserinnen vorgenommen und liegt auf Nachfrage vor.

\section{Schlussfolgerungen}

Wie wir dargestellt haben, trugen Frauen von Anbeginn der Covid-19-Pandemie das Ausfallrisiko des Staates in der Krise, das sich durch die Schließung von Bildungs- und Betreuungseinrichtungen, durch die Mehrbelastungen im Gesundheitswesen und in der kritischen Infrastruktur im Handel ergab. Zumindest während der ersten Phase des Lockdowns 2020 zog sich der Staat in Österreich aus Sorgearbeit um Kinder und Pflegebedürftige in einem hohen Maß zurück und privatisierte Sorgekonflikte und Versorgungskrisen. Soziale Reproduktionsarbeit wurde noch stärker privatisiert beziehungsweise familialisiert und die Versorgungslücke durch die unbezahlte Mehrarbeit von Frauen geschlossen. Die unbezahlte Arbeit von Frauen wurde als Ressource der Krisenbearbeitung einfach vorausgesetzt (Wöhl 2020).

Insgesamt lässt sich festhalten, dass die jetzige Covid-19-Pandemie auf einen bereits in vielen Ländern abgebauten Gesundheitssektor traf, wie z. B. in Spanien. Dies ist die Folge von (Teil-)Privatisierungen im öffentlichen Sektor, der durch die Finanz- und Wirtschaftskrise von 2008ff. besonders vorangetrieben wurde - wie anfangs erläutert. Aber auch in Ländern wie Österreich treffen nicht nur die Folgen der Finanz- und Wirtschaftskrise von 2008 auf Sparmaßnahmen der Regierung vor dem Ausbruch der Covid-19-Pandemie, sondern auch auf einen nicht ausreichend ausgebauten öffentlichen Sektor von Kinder- und Altenbetreuung, der in der jetzigen Gesundheitskrise dann vor allem Frauen trifft, die Kinderbetreuung und die Pflege von Angehörigen zu Hause vermehrt übernommen haben oder übernehmen müssen. Für viele Privathaushalte müssen hier erst Lösungen gefunden werden, wie damit langfristig umgegangen werden kann. Explizit geworden ist dadurch, wie die gleichzeitige Betreuung von Kindern oder von pflegebedürftigen Angehörigen gesellschaftlich überhaupt gewährleistet werden kann. Diese Übernahme der unbezahlten sozialen Reproduktionsarbeit ist jedoch kein Unikum der Covid19-Pandemie, sondern - wie wir mit Verweis auf die Finanz- und Wirtschaftskrise 2008ff. anfangs dargestellt haben - ein Merkmal ökonomischer Krisen und Ausdruck ungleicher Machtverhältnisse, die in Zeiten von Krisen besonders zutage treten. Für die weitere Krisenbearbeitung ist es daher einerseits relevant, wie auf den komplexen Umstand eingegangen werden kann, im Privathaushalt geleistete unbezahlte Mehrfachbelastungen in der sozialen Reproduktionsarbeit auszugleichen, 
und andererseits unbezahlte soziale Reproduktionsarbeit auch in den Budgetbeschlüssen auf nationaler und EU-Ebene in Zukunft einzukalkulieren. Leider zeigt sich, dass sowohl auf nationaler Ebene, wie Femme Fiscale berechnete, Männer von den steuerpolitischen Maßnahmen des österreichischen Konjunkturpakets um ein Viertel mehr profitieren als Frauen. Auch das 750 Milliarden umfassende Wiederaufbau-Programm „Next Generation EU“ auf supranationaler Ebene zielt zwar auf Gleichstellung. Wie der Resilienz- und Recovery-Aufbauplan der jeweiligen Nationalstaaten aber bisher zeigt, werden eher Themen wie Digitalisierung, Umwelt und Verkehr/Infrastruktur aufgegriffen, und ob diese dann wirklich geschlechtergerecht bearbeitet werden, bleibt eine offene Frage.

Die Ökonominnen Elisabeth Klatzer und Azzurra Rinaldi (Klatzer/Rinaldi 2020), die den EU-Plan „Next Generatio EU“ umfassend analysierten, kritisieren, „dass Frauen und Geschlechtergerechtigkeit nur marginal thematisiert werden - obwohl die Covid-Krise zu einer extremen Belastung von Frauen geführt habe. Das Programm habe einen ,Male Employment Bias und lasse gerade jene Branchen außer Acht, in denen besonders viele Frauen arbeiten " (Lichtenberger 2021: 32-33).

Wenn zudem die unbezahlte soziale Reproduktionsarbeit in Privathaushalten im Rahmen des Europäischen Semesters der nationalen Haushaltsplanungen auf nationalstaatlicher Ebene in Zukunft einkalkuliert würde sowie dessen geschlechtsspezifische Effekte berücksichtigt würden, dann könnte eine reale Berechnung der benötigten Mehrausgaben für eine CareÖkonomie des nationalen Staatsbudgets in Zukunft stattfinden, das in bezahlte Pflege und Betreuung investiert und somit auch neue Beschäftigungsverhältnisse schafft, die gesellschaftlich notwendig sind.

\section{Literatur}

Adams-Prassl, A./Boneva, T./Golin, M./Rauh, Ch. (2020): Inequality in the Impact of the Coronovirus Shock: Evidence from Real Time Surveys. IZA Discussion Papers, No. 13183, Institute of Labor Economics (IZA), Bonn.

Allmendinger, J. (2021): Es geht nur gemeinsam! Wie wir endlich Geschlechtergerechtigkeit erreichen. Berlin: Ullstein Verlag.

Alon, T./Doepke, M./Olmstead-Rumsey, J./Tertilt, M. (2020): The impact of Covid-19 on gender equality. Covid Economics, Vetted and Real-Time Papers, The Centre for Economic Policy Research (CEPR), Issue 4, 62-85.
AMS (2021): Spezialauswertung des Arbeitsmarktservice (AMS) Mai 2021 und Bericht Gleichstellungskennzahlen AMS 2020 auf Anfrage von „Diskurs. Das Wissenschaftsnetzt". Online: https://www.diskurs-wissenschaftsnetz. at/wp-content/uploads/2021/o6/PA_Arbeitslosigkeit_ Frauen_DISKURS_01_06_2021.pdf [09.06.2021].

Annesley, A./Scheele, A. (2011): Gender, capitalism and economic crisis: Impact and responses across Europe. Journal of Contemporary European Studies, 19 (3), 335-347.

Arbeiterkammer Wien (2019): Umfrage Gesundheitsberufe: Dringend gebraucht, enorm belastet. Online: https:// wien.arbeiterkammer.at/umfrage-gesundheitsberufe [25.06.2020]

Aulenbacher, B./Dammayr, M. (2014): Krisen des Sorgens. Zur herrschaftsförmigen und widerständigen Rationalisierung und Neuverteilung von Sorgearbeit. In: dies. (Hg.): Für sich und andere sorgen. Krise und Zukunft von Care. Weinheim und Basel: Beltz Juventa, 65-76.

Aulenbacher, B./Bachinger, A./Décieux, F. (2015): Gelebte Sorglosigkeit? Kapitalismus, Sozialstaatlichkeit und soziale Reproduktion am Beispiel des österreichischen „migrant-in-a-family-care“-Modells. Kurswechsel, 1, 6-14.

Aulenbacher, B. (2020): Covid-19 - Warnzeichen oder Weckruf? Über die Sorglosigkeit des Kapitalismus und „Systemrelevanz" des Sorgens. In: Schmidinger, T./Weidenholzer, J. (Hg.): Virenregime. Wie die Corona-Krise unsere Welt verändert. Befunde, Analysen, Anregungen. Wien: Bahoe books, 394-400.

Bakker, I. /Gill, S. (Hg., 2003): Power, Production and Social Reproduction. Basingstoke: Palgrave Macmillan.

Bakker, I. (2007): Social Reproduction and the Constitution of a Gendered Political Economy. New Political Economy, 12 (4), 541-556.

Bauer, L. (2019): Faktencheck: Pflege und Betreuung. Online: https://kompetenz-online.at/2019/04/25/faktencheckpflege-und-betreuung/ [24.06.2020].

Bauer, G. /Rodrigues, R./Leichsenring, K. (2018): Arbeitsbedingungen in der Langzeitpflege aus Sicht der Beschäftigten in Österreich. Eine Untersuchung auf Basis der internationalen NORDCARE-Befragung im Auftrag der Arbeiterkammer Wien. Wien: Europäisches Zentrum für Wohlfahrtspolitik und Sozialforschung. Online: https://www.euro.centre.org/downloads/detail/3288 [21.01.2021].

Benería, L. (1992): The Mexican Debt Crisis, Restructuring the Economy and the Household. In: Benería, L./Feldmann, S. (Hg.): Unequal Burden: Economic Crises, Persistent Poverty and Women's Work. Boulder: Westview Press, 83-104.

Berghammer, C. (2020a): Work-Family-Konflikt in der Corona-Krise. Online: https://viecer.univie.ac.at/coronablog/corona-blog-beitraege/blog25/ [14.06.2020].

Berghammer, C. (2020b): Alles traditioneller? Arbeitsteilung zwischen Männern und Frauen in der Corona-Krise. Online: https://viecer.univie.ac.at/corona-blog/coronablog-beitraege/blog33/ [14.06.2020].
I/IOMENTUM QUARTERLY IIIIIIIIIIIIIIIIIIII 127 
Bina, M./Magnus, A. (2020): Und dann waren wir HeldInnen ... für einmal Klatschen. In: Beigewum (Hg.) COVID-Kaleidoskops. Wie die Krise die Ungleichheit verschärft. Online: http://www.beigewum.at/wp-content/ uploads/Beigewum_CoronaKalaidoskop_Teili_final.pdf [21.01.2021].

Bruff, I./ Wöhl, S. (2016): Implementing Austerity, Disciplining the Household. Masculine Norms of Competitiveness and the Crisis of Social Reproduction in the Eurozone. In: True,J./Hozic', A. (Hg.): Scandalous Economics. Gender and the Politics of Financial Crises, Oxford: Oxford University Press, $92-108$.

Derndorfer, J./Disslbacher, F./Lechinger, V./Mader, K./Six, E. (2021): Home, sweet home? The impact of working from home on the division of unpaid work during the COVID-19 lockdown. INEQ Working Paper Series, 21.

DG Justice (2013): The impact of the economic crisis on the situation of women and men and on gender equality policies - Synthesis Report. Luxembourg: Publication Office of the European Union.

Duma, V./Hajek, K. (2014): Haushaltspolitiken. Feministische Perspektiven auf die Weltwirtschaftskrisen von 1929 und 2008. Österreichische Zeitschrift für Geschichtswissenschaft, 26 (1), 46-74.

European Institute for Gender Equality (2018): Gender budgeting. Mainstreaming gender into the EU budget and macroeconomic policy framework. EIGE Report. Luxembourg: Publications Office of the European Union.

Elias, J./Gunarwardena, S. (Hg., 2013): The Global Political Economy of the Household in Asia. Basingstoke: Palgrave.

Elson, D. (2012): Social Reproduction and the global Crisis. In: Utting, P./Razavi, S. (Hg.): Global Crisis and transformative Social Change. London/New York: Palgrave Macmillan, 63-80.

Farré, L./Fawaz, Y./González, L./Graves, J. (2020): How the COVID-19 Lockdown Affected Gender Inequality in Paid and Unpaid Work in Spain. IZA Discussion Paper No. 13434, Institute of Labor Economics (IZA), Bonn.

Fikenzer, S./Uhe, T./Lavall, D./Rudolph, U./Falz, R./Busse, M./Hepp, P./Laufs, U. (2020): Effects of surgical and $\mathrm{FFP}_{2} / \mathrm{N}_{95}$ face masks on cardiopulmonary exercise capacity. Clinical research in cardiology: official journal of the German Cardiac Society, 109 (12), 1522-1530.

Fukuda-Parr, S./Heintz, J./Seguino, S. (2013): Critical Perspectives on Financial and Economic Crises: Heterodox Macroeconomics meets Feminist Economics. Feminist Economics, 19 (3), 4-31.

González Gago, E./Kirzner, M. S. (2014): Women, Gender Equality and the Economic Crisis in Spain. In: Karamessini, M./Rubery, J. (Hg.): Women and Austerity. The Economic Crisis and the Future for Gender Equality. London: Routledge, 228-247.

Hererra, G. (2012): Starting Over Again? Crisis, Gender and Social Reproduction among Ecuadorian Migrants in Spain. Feminist Economics, 18 (2), 125-148.

Horn, Z. E. (2009): No Cushion to Fall Back On: The Global Economic Crisis and Informal Workers. Synthesis Report,
Inclusive City Studies led by WIEGO, Manchester: WIEGO.

Huke, N./Wöhl, S. (2018): ,Feminisierung der Politik. Soziale Bewegungen gegen Austeritätspolitik in Spanien als Katalysator der politischen Partizipation von Frauen. Momentum Quarterly, 7 (1), 1-52.

Janoch, E. (2020): She told us so. Rapid Gender Analysis: Filling the Data Gap to Build Back Equal. Care International. Online: https://www.care-international.org/files/ files/RGA_SheToldUsSo_9_18_20.pdf [21.01.2021].

Kantola, J./Lombardo, E. (Hg., 2017): Gender and the Crisis in Europe: Politics, Institutions and Intersectionality. Cham: Palgrave.

Karamessini, M./Rubery, J. (Hg., 2014): Women and Austerity. The Economic Crisis and the Future for Gender Equality. London; New York: Routledge.

Klatzer, E./Schlager, Ch. (2011): Europäische Wirtschaftsregierung - eine stille neoliberale Revolution? Kurswechsel, 1, 61-81.

Klatzer, E./Schlager, Ch. (2014): Gender and macroeconomics: economic governance in the European Union reconfiguration of gendered power structures and erosion of gender equality. In: Evans, M./Hemmings, C./Henry, M./ Johnstone, H./Madhok, S./Plomien, A./Wearing, S. (Hg.): The Sage Handbook of Feminist Theory. London: Sage, 483-493.

Klatzer, E./Schlager, Ch. (2020): Losing Grounds: MasculineAuthoritarian Reconfigurations of Power Structures in the European Union. In: Wöhl, S./Springler, E./Pachel, M./Zeilinger, B. (Hg.): The State of the European Union. Fault Lines in European Integration. Wiesbaden: Springer VS, 45-75.

Klatzer, E./Rinaldi, A. (2020): „\#NextGenerationEU“ Leaves Women behind. Gender Impact Assessment of the European Commission Proposals for the EU Recovery Plan, Study commissioned by The Greens/EFA Group in the European Parliament, initiated by Alexandra Geese. MEP June 2020. Online: https://alexandrageese.eu/ wp-content/uploads/2020/o7/Gender-Impact-Assessment-NextGenerationEU_Klatzer_Rinaldi_2020.pdf [18.06.2021].

Kurz-Scherf, I./Scheele, A. (2013): Macht oder ökonomisches Gesetz? Zum Zusammenhang von Krise und Geschlecht. Münster: Westfälisches Dampfboot.

Lichtenberger, H. (2021): Arbeitslos und vielbeschäftigt. Frauenarbeitslosigkeit und Care-Krise in der Pandemie. anschläge. Das Feministische Magazin, 3/2021, 32-33.

Lichtenberger, H./Wöhl, S. (2020): Strukturelle Sorglosigkeit: Die 24-Stunden-Betreuung in der Covid-19-Krise. Femina Politica - Zeitschrift für feministische Politikwissenschaft, 29 (2), 133-134, online: https://doi.org/10.3224/ femina.

Lombardo, E. (2014): Gender mainstreaming and policy responses to the economic crisis: the unintended consequences' of EU and national policymaking on Spanish gender equality. Paper präsentiert an der University of Helsinki, o6. Februar 2014. 
Lombardo, E./León, M. (2015): Políticas de igualdad de género y sociales en España: origen, desarrollo y desmantelamiento en un contexto de crisis económica [Gender equality and social policies in Spain: origin, development and dismantlement in the context of the economic crisis]. Revista de Investigaciones Feministas, 5, 13-35.

Lombardo, E. (2017): The Spanish Gender Regime in the EU Context: Changes and Struggles in Times of Austerity. Gender, Work and Organization, 24 (1), 20-33, online: doi:10.1111/gwao.12148.

Lombardo, E./Alonso, A. (2020): Gender Regime Change in Decentralized States: The Case of Spain. Social Politics 27 (3), 449-466.

López, I./Rodríguez, E. (2011): The Spanish Model. New Left Review, 69 (5), 5-28.

Lux, J./Wöhl, S. (2015): Gender Inequalities in the Crisis of Capitalism: Spain and France Compared In: Bruff, I./ Ebenau, M./May, Ch. (Hg.): New Directions in Comparative Capitalisms Research: Critical and Global Perspectives. London; New York: Palgrave Macmillan, 101-117.

Nagl-Cupal, M./Kolland, F./Zartler, U./Mayer, H./Bittner, M./Koller, M./Parisot, V./Stöhr, D./Bundesministerium für Arbeit, Soziales, Gesundheit und Konsumentenschutz (Hg., 2018): Angehörigenpflege in Österreich. Einsicht in die Situation pflegender Angehöriger und in die Entwicklung informeller Pflegenetzwerke. Wien: Universität Wien. Online: https://broschuerenservice.sozialministerium.at $/$ Home/Download?publicationId $=664$ [14.06.2020].

O’Hagan, A./Klatzer, E. (Hg., 2018): Gender Budgeting in Europe. Development and Challenges. Cham: PalgraveMacmillan.

Schober, M. (2020): Die 24-Stundenbetreuung in Österreich. Eine Analyse der Arbeitsbedingungen von selbstständigen PersonenbetreuerInnen. Masterarbeit JKU Linz. Online: https://epub.jku.at/obvulihs/download/pdf/5267289?ori ginalFilename $=$ true $[21.01 .2020]$.

Serrano, Ch./González Gago, E. (2013): Wirtschaftskrise, Politik, Protest und Geschlecht in Spanien. In: Kurz-Scherf, I./Scheele, A. (Hg.): Macht oder ökonomisches Gesetz? Zum Zusammenhang von Krise und Geschlecht. Münster: Westfälisches Dampfboot, 206-225.

Six, E./Mader, K./Lechinger, V./Disslbacher, F./Derndorfer, J. (2021): Frauen in der Krise? Folgen der Corona-Pandemie für die Verteilung der Sorgearbeit. In: Büro für Frauengesundheit und Gesundheitsziele/Wiener Programm für Frauengesundheit (Hg.): Frauengesundheit und Corona. Wien: Schriftenreihe Frauen ${ }^{\star}$ Gesundheit ${ }^{\star}$ Wien, 1 , 161-170.

SORA (2020): Wie geht es Eltern in der Corona-Pandemie? Online: https://www.sora.at/fileadmin/downloads/projekte/2020_20133_Momentum_Elternbefragung_Corona_Endbericht.pdf [29.06.2021].

Statistik Austria (2019): Personal im Gesundheitswesen. Online: https://www.statistik.at/web_de/statistiken/menschen_und_gesellschaft/gesundheit/gesundheitsver- sorgung/personal_im_gesundheitswesen/index.html [24.6.2020].

Volkshilfe Österreich (2020): Belastet, hilflos, allein gelassen - Umfrage zur Situation von pflegenden Angehörigen armutsbetroffener Demenzerkrankter in der Coronakrise. Online: https://www.ig-pflege.at/downloads/news/2020/ Presseunterlage_Demenzumfrage_12Aug_VOLKSHILFE.pdf?m=1597305393\& [20.01.2021].

von Würzen, B. (2020): Traditionelle Rollenverteilung in Corona-Krise belastet die Frauen. Bielefeld: Bertelsmann Stiftung. Online: https://www.bertelsmann-stiftung.de/ fileadmin/files/user_upload/Spotlight_Rollen_und_ Aufgabenverteilung_bei_Frauen_und_Maennern_in_ Zeiten_von_Corona.pdf [20.1.2021].

Wandler, R. (2020): Corona: Jetzt rächen sich Einsparungen bei Spaniens Spitälern. Online: https://www.derstandard. at/story/2000116253547/corona-jetzt-raechen-sich-einsparungen-bei-spaniens-spitaelern [18.06.2020].

Wirtschaftskammer Österreich (2019): Beschäftigungsstatistik Österreich. Online: https://www.wko.at/service/zahlen-daten-fakten/beschaeftigungsstatistik-oesterreich. html [04.06.2020].

Wöhl, S. (2017): The Gender Dynamics of Financialization and Austerity in the European Union - The Irish Case. In: Kantola, J./Lombardo, E. (Hg.): Gender, Politics, and the Crisis in Europe. Basingstoke: Palgrave Macmillan, 139-159.

Wöhl, S. (2020): Die Bedeutung einer starken öffentlichen Daseinsvorsorge für Geschlechtergerechtigkeit. In: Brauner, R./Müller, B. (Hg.): Wege zur Wohlfahrtstadt. Wirtschafts- und sozialpolitische Überlegungen für eine moderne Kommunalpolitik. Wiener Neustadt: urban future edition, 222-233.

Wöhl, S. (2021): Covid-19. Eine intersektionale Betrachtung. In: Büro für Frauengesundheit und Gesundheitsziele (Hg.): Frauengesundheit und Corona. Wien: Schriftenreihe Frauen ${ }^{\star}$ Gesundheit ${ }^{\star}$ Wien, Nr. 1, 115-120.

Young, B. (2003): Financial Crises and Social Reproduction: Asia, Argentina and Brazil. In: Bakker, I./Gill, S. (Hg.): Power, Production and Social Reproduction. Basingstoke: Palgrave Macmillan, 103-124.
IIIOMENTUM QUARTERLY IIIIIIIIIIIIIIIIIIII

129 